\title{
A tale of two systems: practice patterns of a single group of emergency medical physicians in Taiwan and China
}

I-Anne Huang 1,2,3,4, Tang-Her Jaing ${ }^{2,5}$, Chang-Teng Wu ${ }^{2,5}$, Chee-Jen Chang ${ }^{2,6}$, Shan-Hsuan Hsia ${ }^{2,5}$ and Nicole Huang ${ }^{7^{*}}$ (D)

\begin{abstract}
Background: The quality of pediatric emergency care has been a major concern in health care. Following a series of health system reforms in China, it is important to do this assessment of pediatric emergency care, and to explore potential influences of health care system. This study aimed to compare practice differences in treating children with respiratory illnesses in two emergency department (ED) settings within different health care systems: China and Taiwan.

Methods: A pooled cross-sectional hospital-based study was conducted in two tertiary teaching hospitals in Xiamen, China and Keelung, Taiwan belong to the same hospital chain group. A team of 21 pediatricians rotated between the EDs of the two hospitals from 2009 to 2012. There were 109,705 ED encounters treated by the same team of pediatricians and 6596 visits were analyzed for common respiratory illnesses. Twelve quality measures in process and outcomes of asthma, bronchiolitis and croup were reported. Descriptive statistics and multiple logistic regression models were applied to assess. In order to demonstrate the robustness of our findings, we analyzed the data using an alternative modeling technique, multilevel modeling.

Results: After adjustment, children with asthma presented to the ED in China had a significantly $76 \%$ lower likelihood to be prescribed a chest radiograph, and a 98\% lower likelihood to be prescribed steroids and discharged home than those in Taiwan. Also, children with asthma presented to the ED in China had significantly 7.76 times higher risk to incur 24-72 h return visits. Furthermore, children with bronchiolitis in China (Odds ratio (OR): 0.21; 95\% Confidence interval (Cl): $0.17-0.28$ ) were significantly less likely to be prescribed chest radiograph, but were significantly more likely to be prescribed antibiotics (OR: 2.19; 95\% Cl: 1.46-3.28).

Conclusions: This study illustrated that although high quality care depends on better assessment of physician performance, the delivery of pediatric emergency care differed significantly between these two healthcare systems after holding the care providers the same and adjusting for important patient characteristics. The findings suggest that the features of the health care system may play a significant role.
\end{abstract}

Keywords: Health system, Quality, Practice, Emergency, Children

\footnotetext{
* Correspondence: syhuang@ym.edu.tw

${ }^{7}$ Institute of Hospital and Healthcare Administration, National Yang Ming

University, Room 101, Medical Building II, No.155, Sec. 2, Linong St., Beitou

Dist, Taipei City 112, Taiwan

Full list of author information is available at the end of the article
} 


\section{Background}

Quality of pediatric emergency care has always been a major concern in health care [1-3]. Children constituted one-fifth of emergency department (ED) visits [4]. Variations in the quality of pediatric emergency care and their influencing factors are problems calling for more policy attention [5, 6]. Many factors could have influenced the delivery of pediatric emergency care [7-10]. In terms of individual characteristics, age, race, disease severity, socioeconomic status, and culture are important associating factors [10-12]. In addition, provider characteristics including demographic, training, specialty, patient volume, and adherence to clinical practice guidelines have also been identified as significant relating factors [13, 14]. More importantly, the influences of health system characteristics receive increasing attentions $[5,9,15,16]$. Health system is described as complex adaptive systems by systems thinkers; it means free to respond to different stimuli in unpredictable ways and interconnected with the functions of other parts of a system $[17,18]$. One intuitive approach is to observe the health care delivery in different health systems by controlling similar patients and care provider characteristics; China and Taiwan can serve as a useful and interesting comparison.

China has undergone a major health care reform since 2009. The characteristics of China's health care reform are commonly described as "low level, wide coverage." [19-22] The understanding of the differences in pediatric emergency care with other health systems has been relatively limited. Therefore, using Taiwan, a neighboring society with a well-known comprehensive insurance benefit coverage, as a comparison group may help to provide more insights about the delivery of pediatric emergency care under different health systems.

A large hospital chain group with the same information technology (IT) system and hospital operation in both China and Taiwan offers a unique opportunity to conduct this assessment [23]. Due to the shortage of pediatricians in China, a team of experienced emergency pediatricians from this hospital chain in Taiwan rotate their duties between the hospitals in Taiwan and in China. Therefore, the hospital-based data of this hospital chain during the period of a single team's practice allow us to design a well-controlled pilot study to compare the practice patterns of pediatric emergency care between different health systems in China and Taiwan by maintaining the treating pediatricians and hospitals as a constant variable and then adjusting for comprehensive children's characteristics.

\section{Aim of this study}

Description of the differences between a same group of physicians in two emergency department settings within different systems served as an initial enquiry to establish lines of research into system differences' impact on practitioner management and decision-making.

\section{Methods \\ Study design and setting}

The Chang Gung Memorial Hospital chain group has eight hospitals located in Taiwan and China. The hospital chain under study was first established in Taiwan in 1976 [23]. Two tertiary hospitals, one in Xiamen, China and the other in Keelung, Taiwan, were designated as the study settings. The Xiamen hospital has been in operation since 2008. The two hospitals were selected not only under the same IT and hospital operation systems but also the two cities were very similar in geographic characteristics, of the same language and of the same race, both are located in a suburb, and both overlook the sea, just across the Taiwan Strait, with subtropical climates in East Asia [24, 25]. The two hospitals were also the only tertiary care hospitals within $30 \mathrm{~min}$ travel time in these areas.

A group of pediatric emergency physicians regularly rotated their duty between the emergency departments of the two hospitals during the study period. All of the physicians are trained and board certified physicians in Taiwan. This study had a retrospective design that the physicians were not known the study measures during their practice. The team member staffing level and qualification, the admission wards, and the intensive care units were similar under a hospital chain's operation. The triage classification was based on Taiwan Triage Acuity Scale, standardized in the same IT systems in both hospitals. The emergency pediatricians' income is mainly fixed salary with a proportion of income related to patient volume, but not related to the volume and types of diagnostic testing, prescription, or procedures administered.

A pooled cross-sectional study design was conducted in two tertiary hospitals in Xiamen, China and Keelung, Taiwan to obtain a preliminary estimate of the difference in practice patterns between the same group of physicians in two emergency department settings within different health systems.

\section{Selection of participants}

Figure 1 shows the selection process of our sample. There was a total of 242,335 pediatric emergency visits during the study period. Every physician included in this study had more than 120 patient visits in each hospital branch during the study period. Twenty-one physicians were enrolled in this study and each had managed 3000 to 5000 emergency visits. Only 109,705 ED pediatric visits treated by the same team of 21 physicians were identified. According to previous literatures $[13,14,26,27]$, three groups of pediatric visits were selected for analyses. The first group included children were aged from one to 18 years with a primary or secondary diagnosis of asthma 


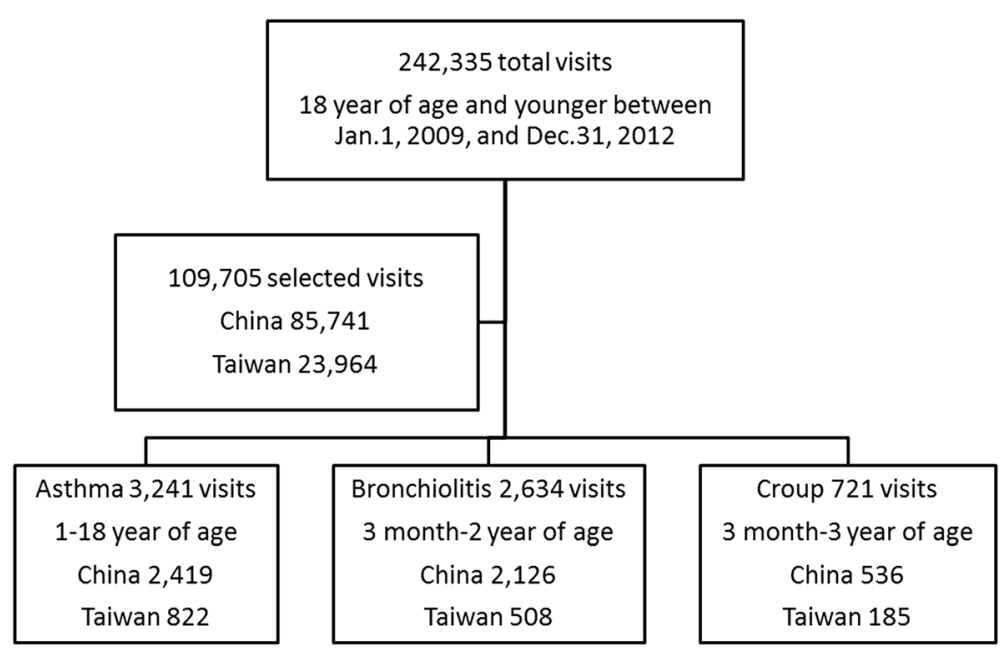

Fig. 1 Study flow chart of sample selection

$(\mathrm{N} 1=3241)$. The second group included children aged from 3 months to 2 years with a primary or secondary diagnosis of bronchiolitis $(\mathrm{N} 2=2634)$, and the third group included children aged between 3 months to 3 years with a primary or secondary diagnosis of croup $(\mathrm{N} 3=721)$.

\section{Health systems}

The quantity of practitioners in a geographic area was four and five physicians per square kilometers in Keelung and Xiamen, respectively [24, 25, 28-30]. There were both 1.9 physicians per thousand people in Keelung and Xiamen [28-30]. The financing is different in the two health systems. Children that visited the ED in Xiamen may or may not have insurance coverage [31,32]. Even with the insurance, people need to pay a large proportion of ED expenses out-of-pocket [32]. The patient's cost sharing was reduced from $79 \%$ in 2009 to $65 \%$ in 2012 [32]. However, in Keelung, Taiwan's National Health Insurance is mandatory and offers comprehensive coverage for emergency services [33]. No co-payment or very minimal copayment is levied for children's emergency care [33]. Despite the differences between the two health systems, they share one feature in common. People can seek care from any provider they like and their access to specialists is not limited or managed by a gatekeeper.

\section{Measures}

As respiratory tract infection is a main reason of pediatric emergency visits, many quality indicators established for pediatric emergency care are specific to pediatric respiratory illnesses, including asthma, bronchiolitis, and croup [13, 14, 27, 34, 35]. Diagnoses were coded using the International Classification of Diseases, Ninth Revision, Clinical Modification (ICD-9-CM) classification in Taiwan, and the International Classification of Disease, Tenth Revision (ICD-10) codes in China. Table 1 lists the ICD-9-CM in Taiwan and ICD-10 codes in China for these diagnoses.

The hospital ED records of the selected pediatric visits between 2009 and 2012 were reviewed in these two tertiary hospitals. Patient-level data included demographics such as age, gender, triage status, season, the timing of the visit, and the insurance status; the clinical information of diagnoses, procedures with imaging, medication, and return visits information were collected from hospital electronic medical records. The standardized processes for asthma, bronchiolitis and croup were the same in the two hospitals during the study period. The protocol for the conduct of this study was reviewed and approved by the Chang Gung Memorial Hospital Institutional Review Board.

According to previous literature, different sets of quality indicators were used for asthma, bronchiolitis, and croup [13, 14, 26, 27, 35]. Four process measures (chest radiograph, the prescription of antibiotics, and steroids in those discharged or admitted) and two outcomes measures (unplanned return visits within $24 \mathrm{~h}$ and 24-72 h) were used for asthma visits of children aged between one to 18 years. Two process measures (chest radiograph and antibiotics prescription) were used for bronchiolitis visits of children aged 3 months to 2 years. Three process measures (chest or lateral neck radiograph, the prescription of steroids, and the patient admitted in ED not receiving steroids) and one outcome measure (unplanned return visit within $24 \mathrm{~h}$ ) were used for croup visits of children aged between 3 months and 3 years.

\section{Statistical analysis}

Descriptive statistics were presented. Chi-square tests and $\mathrm{t}$-tests were used to compare patient characteristics between the Xiamen and Keelung hospitals. Univariate 
Table 1 ICD-9-CM and ICD-10 Codes Used for the Definition of Patient Population

\begin{tabular}{|c|c|c|c|}
\hline & Asthma & Bronchiolitis & Croup \\
\hline \multirow[t]{7}{*}{ ICD-9-CM } & 493.0 & 466 & 464 \\
\hline & 493.01 & 466.0 & 464.0 \\
\hline & 493.02 & 466.1 & 464.2 \\
\hline & 493.1 & 466.11 & 464.20 \\
\hline & 493.10-12 & 466.19 & 464.21 \\
\hline & 493.9 & 487.1 & 464.4 \\
\hline & 493.90-92 & & \\
\hline \multirow[t]{17}{*}{ ICD-10 } & $J 45$ & $J 21$ & J04 \\
\hline & $J 450$ & $J 210$ & J040 \\
\hline & $J 45001-05$ & $J 21051$ & J04001 \\
\hline & $J 45051-53$ & $J 218$ & $J 04002$ \\
\hline & $J 451$ & $J 21851$ & J04004 \\
\hline & $J 45101$ & $J 219$ & $J 04006$ \\
\hline & $J 45151$ & J21901-03 & J042 \\
\hline & $J 45152$ & $\mathrm{~J} 10$ & J04201 \\
\hline & $J 458$ & $J 101$ & $J 05$ \\
\hline & $J 45851$ & $\mathrm{~J} 108$ & J050 \\
\hline & $J 459$ & $\mathrm{~J} 11$ & J05001 \\
\hline & J45901-03 & $\mathrm{J} 111$ & J05051 \\
\hline & $J 45951$ & $J 11101$ & J06 \\
\hline & $J 45952$ & $J 118$ & $J 06001$ \\
\hline & $J 46$ & & J06002 \\
\hline & J46X01 & & J06051 \\
\hline & $J 46 \times 51$ & & \\
\hline
\end{tabular}

and multiple logistic regression models were applied to assess the quality of pediatric emergency care in 12 quality indicators selected in Xiamen while using the Keelung hospital data as a reference. Basically, we selected significantly related factors which have been identified in the existing literature to construct our final multiple logistic regression models $[13,14]$. The comparisons were adjusted for age, gender, triage, season, and time of the visit with 95\% confidence interval for asthma and bronchiolitis groups. For croup, the comparison was adjusted for gender, triage, season, and time of the visit. Since we limited our selection of croup visits to children aged between 3 months to 3 years, the age range was somewhat narrow and within some age categories, the number of children was very small and did not allow for a meaningful comparison.

Furthermore, in the main analyses, triage was reconstructed as a dichotomous variable: triage level 1-3 (resuscitation, emergent, urgent) and triage level 4-5 (semiurgent, non-urgent). In order to examine whether the results remained robust among more urgent and severe visits, we conducted sensitivity analyses by limiting our analyses to the visits with a triage level 1-3 (resuscitation, emergent, urgent). In order to reduce potential biases from clinical variations among the visits, we first limited our analyses among riskier patients with a triage level 1-3. In addition, we conducted another sensitivity analyses to limit the visits with a primary diagnosis of asthma, bronchiolitis and croup. Also, in order to demonstrate the robustness of our findings, we analyzed the data using an alternative modeling technique, multilevel modeling. Due to a possible nested structure of the data (visits nested within providers, we constructed two-level models using PROC GLIMMIX. The first level was visits and the second level was providers. Within the Xiamen sample, quality measures were compared between children both with and without insurance. The level of statistical significance of 0.05 was chosen. All statistical analyses were performed using the statistical software SAS v9.3 (SAS Institute, Inc., Cary, NC).

\section{Results}

Tables 2, 3 and 4 presents distributions of patient characteristics between Xiamen, China and Keelung, Taiwan. Only $44.3 \%, 19.7 \%, 24.4 \%$ of children in the asthma, bronchiolitis, and croup visits in Xiamen had an insurance coverage. More importantly, whereas more than $85 \%$ of pediatric ED visits for asthma (88.0\%), bronchiolitis (89.8\%), or croup (95.1\%) in Keelung were having triage at levels $1-3$, less than $50 \%$ of pediatric ED visits for these conditions (asthma: 18.6\%; bronchiolitis: 29.5\%; croup: $44.6 \%$ ) in Xiamen were having triage at levels 1-3. The percentage of these ED visits resulting in admission in Keelung was 3-4 times higher than that in Xiamen. A significant difference in the timing of ED visits was also observed. More than 50\% of ED visits in Xiamen for these three conditions were made in daytime shifts, but less than $32 \%$ of pediatric ED visits for these conditions in Keelung occurred during daytime shifts.

Table 5 compares the disease-specific quality measures of asthma, bronchiolitis and croup between Xiamen, China and Keelung, Taiwan. In terms of the process indicators for asthma care, while there was only a slight difference in the antibiotic prescription rate between the two settings, the prescription rates of chest radiograph (5.7\%) and steroids (2.0\% at ED discharge) were substantially lower in Xiamen, China than in those in Keelung, Taiwan (chest radiograph: 26.2\%; steroids at ED discharge: $54.1 \%)$. There was only a slight difference in the admitted asthma patients with steroid prescription rates at the ED between the two settings (Xiamen, China: 53.4\%; Keelung, Taiwan: 47.9\%). After adjustment, children with asthma presented to the ED in Xiamen had a significantly $76 \%$ lower likelihood to be prescribed a chest radiograph, and a $98 \%$ lower likelihood to be prescribed steroids and discharged home, but no significant 
Table 2 Sample Characteristics of Pediatric Emergency Department Visits for Asthma by Two Health Systems

\begin{tabular}{|c|c|c|c|c|}
\hline Astma, 1-18 yr & $\begin{array}{l}\text { Overall } \\
N=3241\end{array}$ & $\begin{array}{l}\text { China } \\
N=2419\end{array}$ & $\begin{array}{l}\text { Taiwan } \\
N=822\end{array}$ & $P$ value \\
\hline Age, mean $\pm S D$, yr & $4.7 \pm 2.9$ & $4.0 \pm 2.3$ & $6.9 \pm 3.5$ & $<0.001$ \\
\hline Gender, total n (\%) & & & & 0.053 \\
\hline Male & $2134(65.8 \%)$ & $1570(64.9 \%)$ & $564(68.6 \%)$ & \\
\hline Female & $1107(34.2 \%)$ & 849 (35.1\%) & $258(31.4 \%)$ & \\
\hline Insurance, total n (\%) & & & & $<0.001$ \\
\hline Yes & 1893 (58.4\%) & $1071(44.3 \%)$ & $822(100.0 \%)$ & \\
\hline No & 1348 (41.6\%) & $1348(55.7 \%)$ & $0(0.0 \%)$ & \\
\hline Triage level, total n (\%) & & & & $<0.001$ \\
\hline 1-3: resuscitation, emergent, urgent & $1171(36.1 \%)$ & $448(18.5 \%)$ & $723(88.0 \%)$ & \\
\hline 4-5: semiurgent, nonurgent & 2070 (63.9\%) & 1971 (81.5\%) & 99 (12.0\%) & \\
\hline Season, total n (\%) & & & & 0.772 \\
\hline Spring, Summer & $1394(43.0 \%)$ & $1044(43.2 \%)$ & $350(42.6 \%)$ & \\
\hline Fall, Winter & $1847(57.0 \%)$ & $1375(56.8 \%)$ & $472(56.4 \%)$ & \\
\hline Time of visit, total n (\%) & & & & $<0.001$ \\
\hline Day & $1711(52.8 \%)$ & $1498(61.9 \%)$ & $213(25.9 \%)$ & \\
\hline Night & $1530(47.2 \%)$ & $921(38.1 \%)$ & $609(74.1 \%)$ & \\
\hline Disposition, total n (\%) & & & & $<0.001$ \\
\hline Discharged & 3101 (95.7\%) & 2360 (97.6\%) & 741 (90.2\%) & \\
\hline Admitted & 140 (4.3\%) & 59 (2.4\%) & 81 (9.9\%) & \\
\hline
\end{tabular}

Table 3 Sample Characteristics of Pediatric Emergency Department Visits for Bronchiolitis by Two Health Systems

\begin{tabular}{|c|c|c|c|c|}
\hline Bronchiolitis, 3 m-2y & $\begin{array}{l}\text { Overall } \\
N=2634\end{array}$ & $\begin{array}{l}\text { China } \\
N=2126\end{array}$ & $\begin{array}{l}\text { Taiwan } \\
N=508\end{array}$ & $P$ value \\
\hline Age, mean $\pm S D$, yr & $1.0 \pm 0.6$ & $1.0 \pm 0.6$ & $1.2 \pm 0.6$ & $<0.001$ \\
\hline Gender, total n (\%) & & & & 0.002 \\
\hline Male & $1694(64.3 \%)$ & $1397(65.7 \%)$ & $297(58.5 \%)$ & \\
\hline Female & $940(35.7 \%)$ & $729(34.3 \%)$ & $211(41.5 \%)$ & \\
\hline Insurance, total n (\%) & & & & $<0.001$ \\
\hline Yes & $926(35.2 \%)$ & $418(19.7 \%)$ & $508(100.0 \%)$ & \\
\hline No & 1708 (64.8\%) & $1708(80.3 \%)$ & $0(0.0 \%)$ & \\
\hline Triage level, total n (\%) & & & & $<0.001$ \\
\hline 1-3: resuscitation, emergent, urgent & $1083(41.1 \%)$ & $627(29.5 \%)$ & $456(89.8 \%)$ & \\
\hline 4-5: semiurgent, nonurgent & 1551 (58.9\%) & $1499(70.5 \%)$ & $52(10.2 \%)$ & \\
\hline Season, total n (\%) & & & & 0.006 \\
\hline Spring, Summer & $1400(53.2 \%)$ & $1158(54.5 \%)$ & $242(47.6 \%)$ & \\
\hline Fall, Winter & $1234(46.8 \%)$ & $968(45.5 \%)$ & $266(52.4 \%)$ & \\
\hline Time of visit, total n (\%) & & & & $<0.001$ \\
\hline Day & $1638(62.2 \%)$ & $1477(69.5 \%)$ & $161(31.7 \%)$ & \\
\hline Night & $996(37.8 \%)$ & $649(30.5 \%)$ & $347(68.3 \%)$ & \\
\hline Disposition, total n (\%) & & & & $<0.001$ \\
\hline Discharged & $2351(89.3 \%)$ & 1970 (92.7\%) & 381 (75\%) & \\
\hline Admitted & $283(10.7 \%)$ & 156 (7.3\%) & $127(25 \%)$ & \\
\hline
\end{tabular}


Table 4 Sample Characteristics of Pediatric Emergency Department Visits for Croup by Two Health Systems

\begin{tabular}{|c|c|c|c|c|}
\hline Croup, 3 m-3y & $\begin{array}{l}\text { Overall } \\
N=721\end{array}$ & $\begin{array}{l}\text { China } \\
N=536\end{array}$ & $\begin{array}{l}\text { Taiwan } \\
N=185\end{array}$ & $P$ value \\
\hline Age, mean $\pm S D$, yr & $1.5 \pm 0.8$ & $1.5 \pm 0.8$ & $1.6 \pm 0.8$ & 0.055 \\
\hline Gender, total n (\%) & & & & 0.646 \\
\hline Male & $493(68.4 \%)$ & $364(67.9 \%)$ & $129(69.7 \%)$ & \\
\hline Female & $228(31.6 \%)$ & $172(32.1 \%)$ & $56(30.3 \%)$ & \\
\hline Insurance, total n (\%) & & & & $<0.001$ \\
\hline Yes & $316(43.8 \%)$ & $131(24.4 \%)$ & $185(100.0 \%)$ & \\
\hline No & $405(56.2 \%)$ & $405(75.6 \%)$ & $0(0.0 \%)$ & \\
\hline Triage level, total n (\%) & & & & $<0.001$ \\
\hline 1-3: resuscitation, emergent, urgent & $415(57.6 \%)$ & $239(44.6 \%)$ & $176(95.1 \%)$ & \\
\hline 4-5: semiurgent, nonurgent & $306(42.4 \%)$ & 297 (55.4\%) & $9(4.9 \%)$ & \\
\hline Season, total n (\%) & & & & 0.018 \\
\hline Spring, Summer & $412(57.1 \%)$ & $320(59.7 \%)$ & $92(49.7 \%)$ & \\
\hline Fall, Winter & $309(42.9 \%)$ & $216(40.3 \%)$ & $93(50.3 \%)$ & \\
\hline Time of visit, total n (\%) & & & & $<0.001$ \\
\hline Day & $324(44.9 \%)$ & $269(50.2 \%)$ & $55(29.7 \%)$ & \\
\hline Night & $397(55.1 \%)$ & $267(49.8 \%)$ & $130(70.3 \%)$ & \\
\hline Disposition, total n (\%) & & & & $<0.001$ \\
\hline Discharged & $640(88.8 \%)$ & 501 (93.5\%) & 139 (75.1\%) & \\
\hline Admitted & 81 (11.2\%) & 35 (6.5\%) & 46 (24.9\%) & \\
\hline
\end{tabular}

Table 5 Comparative Data between China and Taiwan in Quality Indicators

\begin{tabular}{|c|c|c|c|c|c|c|}
\hline Asthma, 1-18y, total n (\%) & $\begin{array}{l}\text { China } \\
N=2419\end{array}$ & $\begin{array}{l}\text { Taiwan } \\
N=822\end{array}$ & $\begin{array}{l}\text { Unadjusted Odds Ratio } \\
\text { (95\% Cl) } \\
\text { China vs Taiwan }\end{array}$ & $P$ value & $\begin{array}{l}\text { Adjusted }{ }^{\text {a }} \text { Odds Ratio } \\
(95 \% \text { Cl) } \\
\text { China vs Taiwan }\end{array}$ & $P$ value \\
\hline Radiographs & $137(5.7 \%)$ & $215(26.2 \%)$ & $0.17(0.13-0.21)$ & $<0.001$ & $0.24(0.17-0.33)$ & $<0.001$ \\
\hline Antibiotics & $144(6.0 \%)$ & $60(7.3 \%)$ & $0.80(0.59-1.10)$ & 0.170 & $0.99(0.65-1.52)$ & 0.966 \\
\hline Steroids and discharged home ${ }^{b}$ & $47(2.0 \%)$ & $401(54.1 \%)$ & $0.02(0.02-0.03)$ & $<0.001$ & $0.02(0.01-0.03)$ & $<0.001$ \\
\hline Admitted with steroid in the $\mathrm{ED}^{c}$ & $31(53.4 \%)$ & $39(47.9 \%)$ & $0.26(0.16-0.42)$ & $<0.001$ & $0.45(0.23-0.86)$ & 0.017 \\
\hline Unplanned return visit within $24 \mathrm{~h}$ & $30(1.2 \%)$ & $23(2.8 \%)$ & $0.44(0.25-0.76)$ & 0.002 & $0.61(0.28-1.31)$ & 0.205 \\
\hline Unplanned return visit between 24 and $72 \mathrm{~h}$ & $48(2.0 \%)$ & $2(0.2 \%)$ & $\begin{array}{l}8.30 \\
(2.27-70.63)\end{array}$ & 0.001 & $7.76(1.62-37.17)$ & 0.010 \\
\hline Bronchiolitis, 3 m-2y, total n (\%) & $\begin{array}{l}\text { China } \\
N=2126\end{array}$ & $\begin{array}{l}\text { Taiwan } \\
N=508\end{array}$ & & & & \\
\hline Radiographs & $304(14.3 \%)$ & $267(52.6 \%)$ & $0.15(0.12-0.19)$ & $<0.001$ & $0.21(0.17-0.28)$ & $<0.001$ \\
\hline Antibiotics & $223(10.5 \%)$ & $37(7.3 \%)$ & $1.49(1.04-2.14)$ & 0.030 & $2.19(1.46-3.28)$ & $<0.001$ \\
\hline Croup, 3 m-3y, total n (\%) & $\begin{array}{l}\text { China } \\
N=536\end{array}$ & $\begin{array}{l}\text { Taiwan } \\
\mathrm{N}=185\end{array}$ & & & & \\
\hline Radiographs & $85(15.9 \%)$ & $112(60.5 \%)$ & $0.12(0.08-0.18)$ & $<0.001$ & $0.12(0.08-0.19)$ & $<0.001$ \\
\hline Steroids & $282(52.6 \%)$ & $118(63.8 \%)$ & $0.63(0.45-0.89)$ & 0.008 & $0.93(0.63-1.38)$ & 0.713 \\
\hline Admitted without steroids in $\mathrm{ED}^{c}$ & $14(40.0 \%)$ & $21(45.7 \%)$ & $0.79(0.33-1.94)$ & 0.611 & $0.87(0.33-2.30)$ & 0.774 \\
\hline Unplanned return visit within $24 \mathrm{~h}$ & $17(3.2 \%)$ & $7(3.8 \%)$ & $0.83(0.34-2.04)$ & 0.689 & $0.59(0.20-1.74)$ & 0.338 \\
\hline
\end{tabular}

Taiwan serves as the reference group

${ }^{\mathrm{a}}$ Adjust for age, gender(reference $=$ female), triage(reference $=$ triage level $\left.4-5\right)$, season (reference $=$ spring $\&$ summer $)$ and time of visit(reference $=$ night $)$ in Asthma \& Bronchiolitis

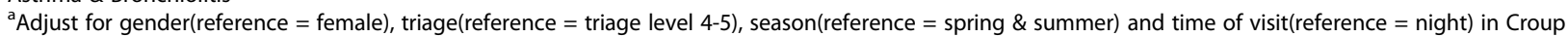
bercentage with the discharge as the denominator

'Percentage with the admitted as the denominator 
difference was observed for either antibiotic prescription or steroids at admission. In terms of outcome measurements, after adjustment, although there was no statistically significant difference in return visit within $24 \mathrm{~h}$, children with asthma presented to the ED in Xiamen had a significantly 7.76 times higher risk to return to the hospital between 24 and $72 \mathrm{~h}$ when compared to those in the Keelung hospital.

Children with bronchiolitis in Xiamen (OR: 0.21; 95\% CI: $0.17-0.28)$ were significantly less likely to be prescribed chest radiograph than those in Keelung, but were significantly more likely to be prescribed antibiotics (OR: 2.19; 95\% CI: 1.46-3.28). In terms of croup care, children in Xiamen were significantly less likely to be prescribed radiographs (OR: 0.12; 95\% CI: 0.08-0.19) and to be admitted (OR: 0.27; 95\% CI: 0.15-0.46) than children in Keelung. In terms of outcome indicators, no significant difference was observed in the return visit within $24 \mathrm{~h}$ between children with croup in two settings.

Furthermore, we also compared 12 quality measures between pediatric ED visits both with and without health insurance in pediatric emergency visits in Xiamen (Table 6). There was no significant difference in quality of ED care observed between children with and without insurance. Finally, as for sensitivity analyses, the results of adopting multilevel models yielded similar significant findings. The results of all other sensitivity anlayses also remained robust.

\section{Discussion}

This study represents a compelling and fascinating glimpse into two pediatric emergency care delivery influenced by different healthcare systems. Although in both Keelung, Taiwan and Xiamen, China, social health care insurance programs are available, the programs are difference in eligibility criteria, extent of benefit coverage, cost-sharing obligations, delivery care system, and reimbursement approaches. Although high quality care depends on better assessment of physician performance, the delivery of pediatric emergency care differed significantly between these two healthcare systems after holding the care providers the same and adjusting for important patient characteristics. Compared to pediatric patients in Keelung, pediatric patients in Xiamen were significantly less likely to receive more expensive diagnostic procedures and medications (i.e. steroid prescription for asthma when discharged home), but more likely to receive potentially inappropriate antibiotics for bronchiolitis, and to incur ER return visits for asthma. Similar potentially inappropriate patterns were also observed for children with insurance in Xiamen.

The pattern of pediatric emergency care for asthma, croup, and bronchiolitis observed in Xiamen, China may be

Table 6 Comparative Data between China with Insurance or not in Quality Indicators

\begin{tabular}{|c|c|c|c|c|c|c|}
\hline Asthma, 1-18y, total n (\%) & $\begin{array}{l}\text { China } \\
\text { no insurance } \\
N=1384\end{array}$ & $\begin{array}{l}\text { China with insurance } \\
N=1071\end{array}$ & $\begin{array}{l}\text { Unadjusted Odds Ratio } \\
(95 \% \mathrm{Cl}) \\
\text { With vs No insurance }\end{array}$ & $P$ value & $\begin{array}{l}\text { Adjusted }{ }^{\mathrm{a}} \text { Odds Ratio } \\
(95 \% \mathrm{Cl}) \\
\text { With vs No insurance }\end{array}$ & $P$ value \\
\hline Radiographs & $81(6.0 \%)$ & $56(5.2 \%)$ & $0.86(0.61-1.23)$ & 0.410 & $0.86(0.60-1.23)$ & 0.410 \\
\hline Antibiotics & $80(5.9 \%)$ & $64(6.0 \%)$ & $1.01(0.72-1.41)$ & 0.966 & $1.05(0.74-1.48)$ & 0.795 \\
\hline Steroids and discharged home ${ }^{b}$ & $24(1.8 \%)$ & $23(2.2 \%)$ & $1.21(0.68-2.16)$ & 0.516 & $1.25(0.69-2.25)$ & 0.465 \\
\hline Admitted with steroid in the $\mathrm{ED}^{\mathrm{c}}$ & $17(47.2 \%)$ & $14(60.9 \%)$ & $1.04(0.51-2.11)$ & 0.920 & $1.90(0.58-6.17)$ & 0.288 \\
\hline Unplanned return visit within $24 \mathrm{~h}$ & $15(1.1 \%)$ & $15(1.4 \%)$ & $1.26(0.61-2.59)$ & 0.525 & $1.25(0.60-2.60)$ & 0.554 \\
\hline $\begin{array}{l}\text { Unplanned return visit between } 24 \\
\text { and } 72 \mathrm{~h}\end{array}$ & $23(1.7 \%)$ & $25(2.3 \%)$ & $1.38(0.78-2.44)$ & 0.271 & $1.34(0.75-2.39)$ & 0.331 \\
\hline Bronchiolitis, 3 m-2y, total n (\%) & $\begin{array}{l}\text { No insurance } \\
N=1708\end{array}$ & $\begin{array}{l}\text { With insurance } \\
N=418\end{array}$ & & & & \\
\hline Radiographs & $245(14.3 \%)$ & $59(14.1 \%)$ & $0.98(0.72-1.33)$ & 0.904 & $1.11(0.81-1.53)$ & 0.514 \\
\hline Antibiotics & $189(11.1 \%)$ & $34(8.1 \%)$ & $0.71(0.49-1.04)$ & 0.080 & $0.71(0.48-1.05)$ & 0.089 \\
\hline Croup, 3 m-3y, total n (\%) & $\begin{array}{l}\text { No insurance } \\
N=405\end{array}$ & $\begin{array}{l}\text { With insurance } \\
N=131\end{array}$ & & & & \\
\hline Radiographs & $68(16.8 \%)$ & $17(13.0 \%)$ & $0.74(0.42-1.31)$ & 0.299 & $0.75(0.42-1.33)$ & 0.317 \\
\hline Steroids & $222(54.8 \%)$ & $60(45.8 \%)$ & $0.70(0.47-1.03)$ & 0.073 & $0.73(0.48-1.09)$ & 0.123 \\
\hline Admitted without steroids in $\mathrm{ED}^{c}$ & $12(40.0 \%)$ & $2(40.0 \%)$ & $0.51(0.05-2.33)$ & 0.534 & $1.04(0.10-10.43)$ & 0.974 \\
\hline Unplanned return visit within $24 \mathrm{~h}$ & $13(3.2 \%)$ & $4(3.1 \%)$ & $0.95(0.30-2.96)$ & 1 & $0.93(0.30-2.92)$ & 0.900 \\
\hline
\end{tabular}

No insurance in China serves as the reference group

${ }^{\mathrm{a}}$ Adjust for age, gender(reference $=$ female), triage(reference $=$ triage level 4-5), season(reference $=$ spring \& summer) and time of visit(reference $=$ night $)$ in Asthma \& Bronchiolitis

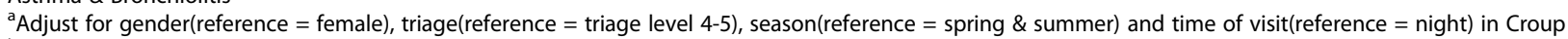

${ }^{\mathrm{b}}$ Percentage with the discharge as the denominator

'Percentage with the admitted as the denominator 
attributable to insufficient health insurance coverage [21, $22,32,36]$, different culture/health beliefs [37-39], and lack of pediatric access to primary care providers, which may worth further investigations. Firstly, unlike the full insurance coverage of emergency care offered to children in Taiwan, on average, a high cost-sharing (i.e. $79 \%$ in 2009 and $65 \%$ in 2012) was associated with emergency services for children in Xiamen, China. According to the existing literature, the prescription of chest radiograph in asthma should be decreased in use and steroids at discharge should be given; the extremely low rates in Xiamen, China were noted $[13,14,26,27,35]$. Substantially lower utilization of the guideline recommended, but relatively expensive diagnostic imaging services may suggest the existence of financial barriers. After limiting the samples to children with the triage level with resuscitation, emergent, and urgent visits, the results remained the same.

One plausible explanation is that only $44.3 \%, 19.7 \%$, and $24.4 \%$ of the children, who had made ER visits for asthma (one - 18 years), bronchiolitis (3 months - 2 years), and croup (3 months- 3 years), respectively, in Xiamen had health insurance coverage. The lack of sufficient insurance poses substantial financial barriers for children to receive quality emergency care. The high percentage of children visiting ER without insurance raises a serious concern. Children without insurance in Xiamen were mostly likely be from migrant families and temporary residents with low socioeconomic status [22,36]. More specifically, even when the children had health insurance in Xiamen, the insurance coverage may not be sufficient in relative low rates of diagnostic tests.

Another interesting finding is that although steroids are recommended for asthma children when discharged home and it is relatively inexpensive in Xiamen, China, a significantly lower likelihood of steroid prescription for asthma when discharged home was observed in Xiamen. According to empirical clinical evidences [27, 38], corticosteroids has been proven effective in controlling symptoms for children with chronic asthma and managing acute onset of pediatric asthma. Appropriate use and application of corticosteroids in front-line pediatric emergency care can help to reduce hospital and emergency room use. Different patterns for prescribing steroids between Xiamen and Keelung may indicate potential differences in culture and people's attitudes toward specific medications in Taiwan and China. Previous studies revealed national and racial disparities in the patient's knowledge about asthma and its management [11, 12, 37-39]. South Asia parents tended to believe that inhaled corticosteroids did more harm than good when compared to the beliefs of Caucasian parents (OR 3.19, CI 1.228.34) [37]. One survey investigated parents of uncontrolled asthma children and found that more parents favored short-acting beta-agonist than inhaled corticosteroids in China (70\%) than in Taiwan (35\%) [38]. Another study in
China also found that parent's fears and concerns about the side effects of steroids was one strongest reason why there was non-adherence to medication among children with asthma [39]. On the other hand, the prescription of antibiotics in bronchiolitis were more commonly prescribed in Xiamen, China than Keelung, Taiwan. To reduce potentially inappropriate prescribing, further qualitative investigations for a deeper understanding the factors driving prescribing behaviors is essential. Parent's beliefs and knowledge shall not be overlooked and may have also influence the health care delivery in the health systems and then change the quality of emergency care received by children.

Thirdly, compared to the ER utilization patterns in Keelung, Taiwan, the utilization of pediatric emergency care in Xiamen had more day-shift visits, more 72-h returns, and more visits with lower triage severity. Whereas community primary care physicians are mostly general practitioners in Xiamen, the findings led to concerns about the availability of community medical resources or accessibility to pediatricians for children in Xiamen. As many of these visits could be appropriately managed by primary care physicians or pediatricians at community settings, it is important to further investigate factors driving parents to seek emergency care for less urgent conditions of their children [40].

A few limitations are notable. First, because our study was hospital-based, performance measurements may be underestimated if the children visited other hospitals for the same episode; we were not able to obtain their utilization of health services in other hospitals. Second, residual confounding may be plausible since information such as parental knowledge, beliefs, or income are not available in our dataset. Third, the study only assessed the quality of three commonly treated respiratory conditions in ER. The results may not be generalizable to other pediatric emergency conditions. Fourth, since our data were limited to one hospital chain group, the results may not be generalizable to other hospitals in China. However, using the detailed information from the hospital information system and the unique staffing rotation feature of this hospital chain allowed us to better control for care providers and patient characteristics. Furthermore, healthcare access and insurance coverage in Xiamen, in general, is better than most of the other cities and regions in China, and so treatment patterns and quality of pediatric care between China and Taiwan may be larger than we observed in our study.

\section{Conclusions}

In summary, this was the first study to illustrate the differences in pediatric emergency care delivery in China provided by a single team's practice under two different 
health care systems using Taiwan as a comparison group. Compared to the situation in Taiwan, serious financial barriers to appropriate diagnostic and treatment procedures still exist among the pediatric population in China, which poses a threat to quality of pediatric emergency care delivery. In addition, parental negative attitudes toward steroids in asthma control and inadequate pediatric resources at community settings may have also influenced the utilization and quality of emergency care in China. Our findings suggest that many pediatric patients may have used ED as a usual source of care for non-urgent conditions. That implies barriers to access for appropriate children's care. The findings may serve as references to future quality improvement efforts in pediatric emergency care in China and Taiwan.

\section{Abbreviations}

Cl: Confidence interval; ED: Emergency department; ICD-10: International Classification of Disease, Tenth Revision; ICD-9-CM: International Classification of Diseases, Ninth Revision, Clinical Modification; IT: Information technology; $\mathrm{N}$ : Numbers; OR: Odds ratio

Acknowledgments

The authors thank the members of the department of Resource Center for Clinical Research, Chang Gung Memorial Hospital at Linkou branch, for the assistance of statistical analyses; and the members of the departments of Management Information System and Medical Affairs, Chang Gung Memorial Hospital at Keelung, Linkou and Xiamen branches, for their assistance with the data acquisition.

\section{Funding}

No funding was received.

\section{Availability of data and materials}

The data that support the findings of this study are available from the Chang Gung Memorial Hospital but restrictions apply to the availability of these data, which were used under license for the current study, and so are not publicly available. Data are however available from the authors upon reasonable request and with permission of the Chang Gung Memorial Hospital.

\section{Authors' contributions}

$\mathrm{IAH}, \mathrm{NH}$, and $\mathrm{SHH}$ designed the data collection instruments, and coordinated and supervised data collection. IAH, CTW, and NH conceptualized and designed the study. THJ and CJC provided statistical advice on study design and analyzed the data. IAH drafted the initial manuscript, and NH made a major revision in writing the manuscript. All authors read and approved the final manuscript. All authors are accountable for all aspects of the manuscript.

\section{Ethics approval and consent to participate}

The protocol for the conduct of this study was reviewed and approved by the Chang Gung Memorial Hospital Institutional Review Board reference number 102-4624B.

Consent to participate in this study is not applicable.

\section{Consent for publication}

Not applicable.

\section{Competing interests}

We declare that we have no conflicts of interest in connection with this paper, except that one of our co-authors, Nicole Huang, is a member of the editorial board (Associate Editor) of this journal.

\section{Publisher's Note}

Springer Nature remains neutral with regard to jurisdictional claims in published maps and institutional affiliations.

\section{Author details}

'Department of Pediatrics, Chang Gung Memorial Hospital, No. 222, Maijin Rd., Keelung, Taiwan. ${ }^{2}$ Graduate Institute of Clinical Medical Sciences, College of Medicine, Chang Gung University, No.259, Wenhua 1st Rd., Guishan Dist, Taoyuan City 333, Taiwan. ${ }^{3}$ Institute of Public Health, National Yang Ming University, No.155, Sec. 2, Linong St., Beitou Dist, Taipei City 112, Taiwan.

${ }^{4}$ Department of Pediatrics, Chang Gung Memorial Hospital, No.123, Xiafei Rd. Haicang Dist, Xiamen City, China. ${ }^{5}$ Department of Pediatrics, Chang Gung Children's Hospital, 5. Fu-hsing St., Guishan Dist, Taoyuan City 333, Taiwan. ${ }^{6}$ Resources Center for Clinical Research, Chang Gung Memorial Hospital, 5. Fu-hsing St., Guishan Dist, Taoyuan City 333, Taiwan. Institute of Hospital and Healthcare Administration, National Yang Ming University, Room 101 Medical Building II, No.155, Sec. 2, Linong St., Beitou Dist, Taipei City 112, Taiwan.

Received: 11 March 2017 Accepted: 7 September 2017

Published online: 11 September 2017

\section{References}

1. Krug SE, Frush K. American Academy of Pediatrics, committee on pediatric emergency medicine. Patient safety in the pediatric emergency care setting. Pediatrics. 2007;120:1367-75.

2. Adirim TA. Emergency medical services for children: thirty years of advancing high-quality emergency care for children. Pediatr Emerg Care. 2015;31:151-6.

3. Institute of Medicine, Committee on the Future of Emergency Care in the United States Health System. Emergnecy Care for Children: growing pains. Washington, DC: National Academies Press; 2006.

4. Wier LM, Yu H, Owens PL, Washington R. Agency for Healthcare Research and Quality. Healthcare Cost and Utilization Project. Overview of children in the emergency department, 2010. http://www.hcup-us.ahrq.gov/reports/ statbriefs/sb157.pdf. Accessed 5 Apr 2016.

5. Chamberlain JM, Krug S, Shaw KN. Emergency care for children in the United States. Health Aff (Millwood). 2013;32:2109-15.

6. Kleinman $L C$, Dougherty D. Assessing quality improvement in health care: theory for practice. Pediatrics. 2013;131(Suppl 1):S110-9.

7. Gausche-Hill M, Ely M, Schmuhl P, et al. A national assessment of pediatric readiness of emergency departments. JAMA Pediatr. 2015;169:527-34.

8. Remick K, Kaji AH, Olson L, et al. Pediatric readiness and facility verification. Ann Emerg Med. 2016;67:320-8.e1.

9. Song Y, Skinner J, Bynum J, Sutherland J, Wennberg JE, Fisher ES. Regional variations in diagnostic practices. N Engl J Med. 2010;363:45-53.

10. Sedaghat AR, Cunningham MJ, Ishman SL. Regional and socioeconomic disparities in emergency department use of radiographic imaging for acute pediatric sinusitis. Am J Rhinol Allergy 2014;28:23-8.

11. James CV, Rosenbaum S. Paying for quality care: implications for racial and ethnic health disparities in pediatric asthma. Pediatrics. 2009;123(Suppl 3): S205-10.

12. Canino G, Koinis-Mitchell D, Ortega AN, McQuaid EL, Fritz GK, Alegria M. Asthma disparities in the prevalence, morbidity, and treatment of Latino children. Soc Sci Med. 2006;63:2926-37.

13. Guttmann A, Weinstein M, Austin PC, Bhamani A, Anderson G. Variability in the emergency department use of discretionary radiographs in children with common respiratory conditions: the mixed effect of access to pediatrician care. CJEM. 2013;15:8-17.

14. Knapp JF, Simon SD, Sharma V. Variation and trends in ED use of radiographs for asthma, bronchiolitis, and croup in children. Pediatrics. 2013;132:245-52.

15. Johnson WG, Rimsza ME. The effects of access to pediatric care and insurance coverage on emergency department utilization. Pediatrics. 2004; 113(3 Pt 1):483-7.

16. Luo X, Liu G, Frush K, Hey LA. Children's Health insurance status and emergency department utilization in the United States. Pediatrics. 2003;112:314-9.

17. Healthy Development The World Bank Strategy for HNP Results Annex L What if a health system?-April 24, 2007. http://siteresources.worldbank.org/ HEALTHNUTRITIONANDPOPULATION/Resources/281627-1154048816360/ AnnexLHNPStrategyWhatisaHealthSystemApril242007.pdf. Accessed 16 Dec 2016.

18. Eyles J, Harris B, Fried J, Govender V, Munyewende P. Endurance, resistance and resilience in the South African health care system: case studies to demonstrate mechanisms of coping within a constrained system. BMC Health Serv Res. 2015. doi: https://doi.org/10.1186/s12913-015-1112-9. 
19. Meng $Q$, Xu L, Zhang $Y$, et al. Trends in access to health services and financial protection in China between 2003 and 2011: a cross-sectional study. Lancet. 2012;379:805-14.

20. Yip WC, Hsiao W, Chen W, Hu S, Ma J, Maynard A. Early appraisal of China's huge and complex health-care reforms. Lancet. 2012;379:833-42.

21. Zhang ZG, Gong X, Luo WJ. A comparative study of urban health insurance system for minors in China. Chinese Health Economics. 2010;29:32-4. [Chinese]. http://d.g.wanfangdata.com.cn/Periodical_zgwsjj201008010.aspx. Accessed 5 Apr 2016.

22. Xiong J, Hipgrave D, Myklebust K, et al. Child health security in China: a survey of child health insurance coverage in diverse areas of the country. Soc Sci Med. 2013;97:15-9.

23. 長庚醫療財團法人維基百科-自由的百科全書. https://zh.m.wikipedia.org/ zh-tw/長庚醫療財團法人. Accessed 5 Apr 2016.

24. 基隆市-維基百科,自由的百科全書. https://zh.wikipedia.org/wiki/ \%E5\%9F\%BA\%E9\%9A\%86\%E5\%B8\%82. Accessed 9 Dec 2016.

25. 廈門市-維基百科,自由的百科全書. https://zh.m.wikipedia.org/wiki/ \%E5\%8E\%A6\%E9\%97\%A8\%E5\%B8\%82. Accessed 9 Dec 2016.

26. Knapp JF, Hall M, Sharma V. Benchmarks for the emergency department care of children with asthma, bronchiolitis, and croup. Pediatr Emerg Care. 2010;26:364-9.

27. Guttmann A, Razzaq A, Lindsay P, Zagorsky B, Anderson GM. Development of measure of the quality of emergency department care for children using a structured panel process. Pediatrics. 2006;118:114-23.

28. National Statistics. http://ebas1.ebas.gov.tw/pxweb2007P/Dialog/varval. asp?ma=Po0201A1A\&ti=\%B0\%F2\%B6\%A9\%A5\%AB\%B2\%CE\%ADp\%A6 \%B3\%F8(\%A6 )\&path=../PXfile17/County17\&lang=9\&strList=L\&strCC=17. Accessed 10 Sept 2017.

29. 統計處首頁 $>$ 衛生福利統計專區 >醫療機構現況及醫院醫療服務量統 計>縣市別. http://www.mohw.gov.tw/CHT/DOS/Statistic.aspx?f_list_no= 312\&fod_list_no=1876. Accessed 9 Dec 2016.

30. 廈門統計局. http://www.stats-xm.gov.cn/tjzl/tjgb/. Accessed 9 Dec 2016.

31. 全民醫保「廈門模式」全國競仿. 廈門新視線香港文匯報: 廈門新視 線(38): July 1, 2009. http://paper.wenweipo.com/2009/07/01/zt0907010091. htm. Accessed 10 Sept 2017.

32. 未成年人医疗保险政策业务指南 - 厦门地方税务局www.xm-I-tax.gov.cn/ downfiles/clj|x/xmds9491_2.doc. Accessed 5 Apr 2016.

33. Ho Chan WS. Taiwan's Healthcare report 2010. EPMA J. 2010;1:563-85.

34. Liao $P$, Ku M, Lue $K$, Sun $H$. Respiratory tract infection is the major cause of the ambulatory visits in children. Ital J Pediatr. 2011;37:43-7.

35. Knapp JF, Simon SD, Sharma V. Quality of care for common pediatric respiratory illnesses in United States emergency departments: analysis of 2005 National Hospital Ambulatory Medical Care Survey data. Pediatrics. 2008;122:1165-70

36. Lu M, Zhang J, Ma J, Li B, Quan H. Child health insurance coverage: a survey among temporary and permanent residents in shanghai. BMC Health Serv Res. 2008;8:238-46

37. Smeeton N, Rona RJ, Gregory J, White P, Morgan M. Parental attitudes towards the management of asthma in minority ethnic communities. Arch Dis Child. 2007:92:1082-7.

38. Wong GW, Kwon N, Hong JG, Hsu JY, Gunasekera KD. Pediatric asthma control in Asia: phase 2 of the asthma insights and reality in Asia-Pacific (AIRIAP 2) survey. Allergy. 2013;68:524-30.

39. Zhao J, Shen $K$, Xiang $L$, et al. The knowledge, attitudes and practices of parents of children with asthma in 29 cities of China: a multi-center study. BMC Pediatr. 2013;13:20-5

40. Benahmed N, Laokri S, Zhang WH, et al. Determinants of nonurgent use of the emergency department for pediatric patients in 12 hospitals in Belgium. Eur J Pediatr. 2012:171:1829-37.

\section{Submit your next manuscript to BioMed Central and we will help you at every step:}

- We accept pre-submission inquiries

- Our selector tool helps you to find the most relevant journal

- We provide round the clock customer support

- Convenient online submission

- Thorough peer review

- Inclusion in PubMed and all major indexing services

- Maximum visibility for your research

Submit your manuscript at www.biomedcentral.com/submit

) Biomed Central 